\title{
Psychiatric onset of multiple sclerosis
} Georgia Deretzi ${ }^{* 1}$, Konstantinos Petsanis ${ }^{1}$, George Floros ${ }^{1}$, Evaggelos Papamihalis ${ }^{1}$, Modestos Gounaris ${ }^{1}$, Aikaterini Dimakopoulou ${ }^{1}$, Dimitrios Sevris $^{2}$ and Nikolaos Grigoriadis ${ }^{3}$

\author{
Address: ${ }^{1}$ Neurology Department, Hippokration Hospital, Thessaloniki, Greece, ${ }^{2} \mathrm{~B}$ Acute Ward, Psychiatric Hospital of Thessaloniki, Greece and \\ ${ }^{3}$ 2nd Department of Neurology, AHEPA University Hospital, Thessaloniki, Greece \\ * Corresponding author
}

from International Society on Brain and Behaviour: 3rd International Congress on Brain and Behaviour

Thessaloniki, Greece. 28 November - 2 December 2007

Published: 17 April 2008

Annals of General Psychiatry 2008, 7(Suppl I):SI39 doi:I0.II86/I744-859X-7-SI-SI 39

This abstract is available from: http://www.annals-general-psychiatry.com/content/7/SI/SI39

(C) 2008 Deretzi et al.; licensee BioMed Central Ltd.

\section{Background}

Although psychiatric dysfunctions are well recognized throughout the course of definite multiple sclerosis (MS), initial presentation of the disease with predominant psychiatric symptoms has rarely been reported.

\section{Materials and methods}

A detailed report of four cases of young women whose atypical first presentation of MS led to them being initially diagnosed with psychiatric diseases. Emphasis is placed on differential diagnosis and timely identification of similar cases.

\section{Results}

Four women (aged 30, 32, 33 and 38 years) out of 298 consecutive patients with definite MS, exhibited a psychiatric syndrome at initial presentation without other symptoms or signs suggestive for MS. Brain CT scans were normal. Three out of the four patients underwent psychiatric hospitalizations. Two of the patients were initially diagnosed with manic-depressive disorder, one with major depression and one with psychosis. MS diagnosis was delayed and established within one to eight years following the initial psychiatric presentation. All patients underwent brain and spinal MRI and brain SPECT. Patients fulfilled the recently revised McDonald diagnostic criteria of MS. Patients were followed for three to up to ten years thereafter. All of them gradually developed characteristic physical signs, with concomitant disabilities.

\section{Conclusions}

MS should be considered in the differential diagnosis of a psychiatric disorder even in the absence of typical neurological signs. Careful relevant clinical and laboratory investigation as well as neuroimaging techniques should be performed, especially in cases of previously healthy young patients who present with atypical psychiatric manifestations. Early diagnosis may be particularly important in view of the emerging disease-modifying treatments. 